TRANSACTIONS OF THE

AMERICAN MATHEMATICAL SOCIETY

Volume 360, Number 11, November 2008, Pages 6131-6147

S 0002-9947(08)04506-6

Article electronically published on June 26, 2008

\title{
APPLICATIONS OF THE FUGLEDE-KADISON DETERMINANT: SZEGÖ'S THEOREM AND OUTERS FOR NONCOMMUTATIVE $H^{p}$
}

\author{
DAVID P. BLECHER AND LOUIS E. LABUSCHAGNE
}

\begin{abstract}
We first use properties of the Fuglede-Kadison determinant on $L^{p}(M)$, for a finite von Neumann algebra $M$, to give several useful variants of the noncommutative Szegö theorem for $L^{p}(M)$, including the one usually attributed to Kolmogorov and Krein. As an application, we solve the longstanding open problem concerning the noncommutative generalization, to Arveson's noncommutative $H^{p}$ spaces, of the famous 'outer factorization' of functions $f$ with $\log |f|$ integrable. Using the Fuglede-Kadison determinant, we also generalize many other classical results concerning outer functions.
\end{abstract}

\section{INTRODUCTION}

It has long been of great importance to operator theorists and operator algebraists to find noncommutative analogues of the classical 'inner-outer factorization' of analytic functions. We recall some classical results: If $f \in L^{1}$ with $f \geq 0$, then $\int \log f>-\infty$ iff $f=|h|$ for an outer $h \in H^{1}$ (iff $f=|h|^{p}$ for an outer $\bar{h} \in H^{p}$ ). We will call this the Riesz-Szegö theorem since it is often attributed to one or the other of these authors. If $f \in L^{1}$ with $\int \log |f|>-\infty$, then $f=u h$, where $u$ is unimodular and $h$ is outer. Moreover, outer functions may be defined in terms of a simple equation involving $\int \log |f|$. Such results are usually treated as consequences of the classical Szegö theorem, which is really a distance formula in terms of the entropy $\exp \left(\int \log |f|\right)$, and which in turn is intimately related to the Jensen inequality (see e.g. [17]). In the noncommutative situation one wishes, for example, to find conditions on a positive operator $T$ which imply that $T=|S|$ for an operator $S$ which is in a 'noncommutative Hardy class', or, even better, which is 'outer' in some sense. There are too many such results in the literature to attempt a listing of them (see e.g. [26, p. 1495]). Indeed this is an active and important research area which has links to many exciting parts of mathematics. Interestingly, central parts of this topic still seem to be poorly understood. As an example of this, we cite the main and now classical result of [9], concerning a Riesz-Szegö like factorization of a class of $B(H)$-valued functions on the unit interval, which has resisted generalization in some important directions. In our paper we generalize the classical

Received by the editors September 20, 2006 and, in revised form, February 22, 2007.

2000 Mathematics Subject Classification. Primary 46L51, 46L52, 47L75; Secondary 46J15, 46K50, 47L 45 .

The first author was partially supported by grant DMS 0400731 from the National Science Foundation.

The second author was partially supported by a National Research Foundation Focus Area Grant. 
results above to the noncommutative $H^{p}$ spaces associated with Arveson's remarkable subdiagonal algebras [1. Our generalization solves an old open problem (see the discussion in [26, lines 8-12, p. 1497] and [21, p. 386]). The approach which we take has been unavailable until now (since it relies ultimately on the recent solution in [20] of a 40 year old open problem from [1]). Moreover, the approach is very faithful to the original classical function theoretic route (see e.g. [17]), proceeding via noncommutative Szegö theorems.

In the last several years we have attempted to demonstrate that all the results in (a particular survey [30] of) the 'generalized $H^{p}$-theory' for abstract function algebras from the 1960s extend, in an extremely complete and literal fashion, to the noncommutative setting of Arveson's subdiagonal subalgebras of von Neumann algebras [1. This may be viewed as a very natural merging of generalized Hardy space, von Neumann algebra, and noncommutative $L^{p}$ space, techniques. See our recent survey [7] for an overview of this work. The present paper completes the noncommutative extension of the basic Hardy space theory. As posited by Arveson, one should use the Fuglede-Kadison determinant $\Delta(a)=\exp (\tau(\log |a|))$, where $\tau$ is a trace, as a natural replacement in the noncommutative case for the quantity $\int \log f$ above. We use properties of the Fuglede-Kadison determinant to give several useful variants of the noncommutative Szegö theorem for $L^{p}(M)$, including the one usually attributed to Kolmogorov and Krein. As applications, we generalize the noncommutative Jensen inequality, and generalize many of the classical results concerning outer functions, to the noncommutative $H^{p}$ context. Some of these will be described in more detail later in this introduction.

We now review some of the definitions and notation, although we strongly suggest that the reader glance through our survey article [7] first for background, motivation, history, etc. For a set $\mathcal{S}$, we write $\mathcal{S}_{+}$for the set $\{x \in \mathcal{S}: x \geq 0\}$. We assume throughout that $M$ is a von Neumann algebra possessing a faithful normal tracial state $\tau$. The existence of such a $\tau$ implies that $M$ is a so-called finite von Neumann algebra, and that if $x^{*} x=1$ in $M$, then $x x^{*}=1$ too. Indeed, for any $a, b \in M, a b$ will be invertible precisely when $a$ and $b$ are separately invertible. We will also need to use a well-known fact about inverses of an unbounded operator $T$, and in our case $T$ will be positive, selfadjoint, closed, and densely defined. We recall that $T$ is bounded below if for some $\lambda>0$ one has $\|T(\eta)\| \geq \lambda\|\eta\|$ for all $\eta \in \operatorname{dom}(T)$. This is equivalent to demanding that $|T| \geq \epsilon 1$ for some $\epsilon>0$, and of course in this case, $|T|$ has a bounded positive inverse.

A (finite maximal) subdiagonal subalgebra of $M$ is a weak* closed unital subalgebra $A$ of $M$ such that if $\Phi$ is the unique conditional expectation guaranteed by [31, p. 332] from $M$ onto $A \cap A^{*} \stackrel{\text { def }}{=} \mathcal{D}$ which is trace preserving (that is, $\tau \circ \Phi=\tau$ ), then

$$
\Phi\left(a_{1} a_{2}\right)=\Phi\left(a_{1}\right) \Phi\left(a_{2}\right), \quad a_{1}, a_{2} \in A .
$$

One also must impose one further condition on $A$. There is a choice of at least eight equivalent, but quite different looking, conditions [7; Arveson's original one (see also [10]) is that $A+A^{*}$ is weak* dense in $M$. In the classical function algebra setting [30, one assumes that $\mathcal{D}=A \cap A^{*}$ is one dimensional, which forces $\Phi=\tau(\cdot) 1$. If in our setting this is the case, then we say that $A$ is antisymmetric.

The simplest example of a maximal subdiagonal algebra is the upper triangular matrices $A$ in $M_{n}$. Here $\Phi$ is the expectation onto the main diagonal. There are 
much more interesting examples from free group von Neumann algebras, TomitaTakesaki theory, etc. (see, e.g., [1, 34, 21]). On the other end of the spectrum, $M$ itself is a maximal subdiagonal algebra (take $\Phi=I d$ ). It is therefore remarkable that so much of the classical $H^{p}$ theory does extend to all maximal subdiagonal algebras. However the reader should not be surprised to find one or two results below which do impose restrictions on the size of $\mathcal{D}$.

By analogy with the classical case, we set $A_{0}=A \cap \operatorname{Ker}(\Phi)$ and set $H^{p}$ or $H^{p}(A)$ to be $[A]_{p}$, the closure of $A$ in the noncommutative $L^{p}$ space $L^{p}(M)$, for $p \geq 1$. More generally we write $[\mathcal{S}]_{p}$ for this closure of any subset $\mathcal{S}$. We will often view $L^{p}(M)$ inside $\widetilde{M}$, the set of unbounded, but closed and densely defined, operators on $H$ which are affiliated to $M$. In the present context this is a *-algebra with respect to the 'strong' sum and product (see Theorem I.28 and the example following it in 32 ). We order $\widetilde{M}$ by its cone of positive (selfadjoint) elements. The trace $\tau$ extends naturally to the positive operators in $\widetilde{M}$. If $1 \leq p<\infty$, then $L^{p}(M, \tau)=\left\{a \in \widetilde{M}: \tau\left(|a|^{p}\right)<\infty\right\}$, equipped with the norm $\|\cdot\|_{p}=\tau\left(|\cdot|^{p}\right)^{1 / p}$ (see, e.g., 25, 11, 32, 26]). We abbreviate $L^{p}(M, \tau)$ to $L^{p}(M)$.

Arveson's Szegö formula is

$$
\Delta(h)=\inf \left\{\tau\left(h|a+d|^{2}\right): a \in A_{0}, d \in \mathcal{D}, \Delta(d) \geq 1\right\}
$$

for all $h \in L^{1}(M)_{+}$. Here $\Delta$ is the Fuglede-Kadison determinant, originally defined on $M$ by $\Delta(a)=\exp \tau(\log |a|)$ if $|a|>0$, and otherwise, $\Delta(a)=\inf \Delta(|a|+\epsilon 1)$, the infimum taken over all scalars $\epsilon>0$ (see [12, 1). We will discuss this determinant in more detail in Section 2. Unfortunately, the just-stated noncommutative Szegö formula, and the (no doubt more important) associated Jensen's inequality

$$
\Delta(\Phi(a)) \leq \Delta(a), \quad a \in A,
$$

resisted proof for nearly 40 years, although Arveson did prove them in his extraordinary original paper [1] for the examples that he was most interested in. In 2004, the second author proved in 20] that all maximal subdiagonal algebras satisfy these formulae. Settling this old open problem opened up the theory and, in particular, enabled the present work.

An element $h \in H^{p}$ is said to be outer if $1 \in[h A]_{p}$. This definition is in line with e.g. Helson's definition of outers in the matrix valued case he considers in [15]. We now state a sample of our results about outers. For example, we are able to improve on the factorization theorems from e.g. [5. Section 3] in several ways: namely we show that if $f \in L^{p}(M)$ with $\Delta(f)>0$, then $f$ may be essentially uniquely factored as $f=u h$ with $u$ unitary and $h$ outer. There is a much more obvious converse to this, too. Moreover we now have an explicit formula for the $u$ and $h$. We refer to a factorization $f=u h$ of this form as a Beurling-Nevanlinna factorization. It follows that in this case if $f \geq 0$, then $f=|h|$ with $h$ outer. This gives a solution to the problem posed in [26, Remark after Theorem 8.1], and in [21, p. 386]. If $h \in H^{p}$ and $h$ is outer, then $\Delta(h)=\Delta(\Phi(h))$. Moreover, a converse is true: if $\Delta(h)=\Delta(\Phi(h))>0$, then $h$ is outer. It follows that under some restrictions on $\mathcal{D}=A \cap A^{*}, h$ is outer iff $\Delta(h)=\Delta(\Phi(h))>0$.

Some historical remarks: there are many factorization theorems for subdiagonal algebras in the literature (see, e.g., [1, 21, 28, 22, 26]), but as far as we know there are 
no noncommutative factorization result 1 involving outers or the Fuglede-Kadison determinant. We mention for example Arveson's original factorization result from [1], or Marsalli and West's Riesz factorization of any $f \in H^{1}$ as a product $f=g h$ with $g \in H^{p}, h \in H^{q}, \frac{1}{p}+\frac{1}{q}=1$. Some also require rather stronger hypotheses, such as $f^{-1} \in L^{2}(M)$ (see e.g. [21]).

Another important historical remark is that the commutative case of most of the topics in our paper was settled in [23. While this paper certainly gave us motivation to persevere in our endeavor, we follow completely different lines, and indeed the results work out rather differently too. In particular, the quantity $\tau(\exp (\Phi(\log |f|)))$, which plays a central role in most of the results in 23], seems to us to be unrelated to outers or factorization in the noncommutative setting. In passing, we remark that numerical experiments do seem to confirm the existence of a Jensen inequality $\tau(\exp (\Phi(\log |a|))) \geq \tau(|\Phi(a)|)$ for subdiagonal algebras. However, even if correct, it is not clear how this might be useful to the present work. We therefore defer such considerations to a future investigation.

Finally, we remark that there are many other, more recent, generalizations of $H^{\infty}$, based around multivariable analogues of the Sz.-Nagy-Foiaş model theory for contractions. In essence, the unilateral shift is replaced by left creation operators on some variant of Fock space. Many prominent researchers are currently intensively pursuing these topics; they are very important and are evolving in many directions. Although these theories also contain variants of Hardy space theory, they are quite far removed from subdiagonal algebras. For example, if one compares Popescu's theorem of Szegö type from [27, Theorem 1.3] with the Szegö theorem for subdiagonal algebras discussed here, one sees that they are only related in a very formal sense.

\section{Properties of the Fuglede-Kadison determinant}

The Fuglede-Kadison determinant $\Delta$, and its amazing properties, is perhaps the main tool in the noncommutative $H^{p}$ theory. In [11, Fuglede and Kadison study the determinant as a function on $M$. In the next paragraph we will define it for elements of $L^{q}(M)$ for any $q>0$. In fact, as was pointed out to us by Quanhua $\mathrm{Xu}, \mathrm{L} . \mathrm{G}$. Brown investigated the determinant and its properties in the early 1980s, on a much larger class than $L^{q}(M)$ (see 8]); indeed recently Haagerup and Schultz have thoroughly explicated the basic theory of this determinant for a very general class of $\tau$-measurable operators (see [14]) as part of Haagerup's amazing attack on the invariant subspace problem relative to a finite von Neumann algebra.

For our purposes, we will define the Fuglede-Kadison determinant for an element $h \in L^{q}(M)$, for any $q>0$, as follows. We set $\Delta(h)=\exp \tau(\log |h|)$ if $|h|>\epsilon 1$ for some $\epsilon>0$, and otherwise, $\Delta(h)=\inf \Delta(|h|+\epsilon 1)$, the infimum taken over all scalars $\epsilon>0$. To see that this is well-defined, we adapt the argument in the third paragraph of [5, Section 2], making use of the Borel functional calculus for unbounded operators applied to the inequality

$$
0 \leq \log t \leq \frac{1}{q} t^{q}, \quad t \in[1, \infty) .
$$

\footnotetext{
${ }^{1}$ With the exception of the factorization results in our previous paper [5], which are in many senses improved upon here.
} 
Notice that for any $0<\epsilon<1$, the function $\log t$ is bounded on $[\epsilon, 1]$. So given $h \in L^{q}(M)_{+}$with $h \geq \epsilon 1$, it follows that $(\log h) e_{[0,1]}$ is similarly bounded. Moreover the previous centered equation ensures that $0 \leq(\log h) e_{[1, \infty)} \leq \frac{1}{q} h^{q} e_{[1, \infty)} \leq \frac{1}{q} h^{q}$. Here $e_{[0, \lambda]}$ denotes the spectral resolution of $h$. Thus if $h \in L^{q}(M)$ and $h \geq \epsilon$, then $\log h \in L^{1}(M)$.

The following are the basic properties of this extended determinant which we shall need. Full proofs may be found in [14, which are valid for a very general class of unbounded operators (see also [7] for another (later) proof for the $L^{p}(M)$ class). We will often use these results silently in the next few sections.

Theorem 2.1. If $p>0$ and $h \in L^{p}(M)$, then

(1) $\Delta(h)=\Delta\left(h^{*}\right)=\Delta(|h|)$.

(2) If $h \geq g$ in $L^{p}(M)_{+}$, then $\Delta(h) \geq \Delta(g)$.

(3) If $h \geq 0$, then $\Delta\left(h^{q}\right)=\Delta(h)^{q}$ for any $q>0$.

(4) $\Delta(h b)=\Delta(h) \Delta(b)=\Delta(b h)$ for any $b \in L^{q}(M)$ and any $q>0$.

\section{SzEGÖ's FORMULA REVISITED}

Throughout this section, $A$ is a maximal subdiagonal algebra in $M$. We consider versions of Szegö's formula valid in $L^{p}(M)$ rather than $L^{2}(M)$. We will also prove a generalized Jensen inequality and show that the classical Verblunsky/KolmogorovKrein strengthening of Szegö's formula extends even to the noncommutative context.

It is proved in [6] that for $h \in L^{1}(M)_{+}$and $1 \leq p<\infty$, we have

$$
\Delta(h)=\inf \left\{\tau\left(h|a+d|^{p}\right): a \in A_{0}, d \in \mathcal{D}, \Delta(d) \geq 1\right\} .
$$

We now prove some perhaps more useful variants of this formula.

Lemma 3.1. If $h \in L^{q}(M)_{+}$and $0<p, q<\infty$, we have

$$
\Delta(h)=\inf \left\{\tau\left(\left|h^{\frac{q}{p}} b\right|^{p}\right)^{\frac{1}{q}}: b \in M_{+}, \Delta(b) \geq 1\right\}=\inf \left\{\tau\left(\left|b h^{\frac{q}{p}}\right|^{p}\right)^{\frac{1}{q}}: b \in M_{+}, \Delta(b) \geq 1\right\} .
$$

The infimums are realized on the commutative von Neumann subalgebra $M_{0}$ generated by $h$ and are unchanged if, in addition, we also require $b$ to be invertible in $B$.

Proof. That the two infimums in the displayed equation are equal follows from the fact that $\|x\|_{p}=\left\|x^{*}\right\|_{p}$ for $x \in L^{p}(M)$ (see [1]). Thus we just prove the first equality in that line.

For $b \in M_{+}, \Delta(b) \geq 1$, we have by Theorem 2.1 (3) that

$$
\Delta\left(\left|h^{q / p} b\right|^{p}\right)=\Delta\left(\left|h^{q / p} b\right|\right)^{p}=\Delta\left(h^{q / p} b\right)^{p} .
$$

Consequently, using facts from Theorem 2.1 again, we have

$$
\tau\left(\left|h^{q / p} b\right|^{p}\right) \geq \Delta\left(\left|h^{q / p} b\right|^{p}\right)=\left[\Delta\left(h^{q / p}\right) \Delta(b)\right]^{p} \geq \Delta\left(h^{q / p}\right)^{p}=\Delta(h)^{q} .
$$

To complete the proof, it suffices to find, given $\epsilon>0$, an invertible $b$ in $\left(M_{0}\right)_{+}$, the von Neumann algebra generated by $h$ (see the first paragraph of Section 2), with $\Delta(b) \geq 1$ and $\tau\left(\left|h^{\frac{q}{p}} b\right|^{p}\right)^{\frac{1}{q}}<\Delta(h)+\epsilon$. But for any $b \in\left(M_{0}\right)_{+}$we have $\left|h^{q / p} b\right|^{p}=h^{q} b^{p}$ by commutativity, and then the result follows from an analysis of Arveson's original definition of $\Delta(h)$ (see (2.1) in [4]). In particular since $\Delta\left(h^{q}\right)=\inf \left\{\tau\left(h^{q} b^{p}\right): b \in\right.$ $\left.\left(M_{0}\right)_{+}, \Delta(b) \geq 1\right\}$ by [4, Theorem 2.1], an application of Theorem 2.1 (3) ensures that $\Delta(h)=\inf \left\{\tau\left(h^{q} b^{p}\right)^{\frac{1}{q}}: b \in\left(M_{0}\right)_{+}, \Delta(b) \geq 1\right\}$. 
Corollary 3.2. If $h \in L^{q}(M)_{+}$and $0<p, q<\infty$, we have

$$
\begin{aligned}
\Delta(h) & =\inf \left\{\tau\left(\left|h^{\frac{q}{p}} a\right|^{p}\right)^{\frac{1}{q}}: a \in A, \Delta(\Phi(a)) \geq 1\right\} \\
& =\inf \left\{\tau\left(\left|a h^{\frac{q}{p}}\right|^{p}\right)^{\frac{1}{q}}: a \in A, \Delta(\Phi(a)) \geq 1\right\} .
\end{aligned}
$$

The infimums are unchanged if we also require a to be invertible in $A$, or if we require $\Phi(a)$ to be invertible in $\mathcal{D}$.

Proof. That the two infimums in the displayed equation are equal follows from the fact that $\|x\|_{p}=\left\|x^{*}\right\|_{p}$ for $x \in L^{p}(M)$ (see [11]), and by replacing $A$ with $A^{*}$, which is also subdiagonal. Thus we just prove the first equality in that line.

For $a \in A, \Delta(\Phi(a)) \geq 1$ we have

$$
\tau\left(\left|h^{\frac{q}{p}} a\right|^{p}\right)^{\frac{1}{q}}=\tau\left(\left|a^{*} h^{\frac{q}{p}}\right|^{p}\right)^{\frac{1}{q}}=\tau\left(|| a^{*}\left|h^{\frac{q}{p}}\right|^{p}\right)^{\frac{1}{q}} \geq \Delta(h),
$$

by Lemma 3.1, since $\Delta\left(\left|a^{*}\right|\right)=\Delta\left(a^{*}\right)=\Delta(a) \geq \Delta(\Phi(a)) \geq 1$ (using Jensen's inequality). Thus $\Delta(h)$ is dominated by the first infimum. On the other hand, by the previous result there is an invertible $b \in M_{+}$with $\Delta(b) \geq 1$ and $\tau\left(\left|b h^{\frac{q}{p}}\right|^{p}\right)^{\frac{1}{q}}<$ $\Delta(h)+\epsilon$. By factorization, we can write $b=\left|a^{*}\right|$ for an invertible $a$ in $A$, and by Jensen's formula [1, 20] we have $\Delta(\Phi(a))=\Delta(a)=\Delta\left(a^{*}\right)=\Delta(b) \geq 1$. Then

$$
\tau\left(\left|h^{\frac{q}{p}} a\right|^{p}\right)^{\frac{1}{q}}=\tau\left(\left|a^{*} h^{\frac{q}{p}}\right|^{p}\right)^{\frac{1}{q}}=\tau\left(\left|b h^{\frac{q}{p}}\right|^{p}\right)^{\frac{1}{q}}<\Delta(h)+\epsilon .
$$

We leave the remaining details to the reader.

Corollary 3.3 (Generalized Jensen inequality). Let $A$ be a maximal subdiagonal algebra. For any $h \in H^{1}$ we have $\Delta(h) \geq \Delta(\Phi(h))$.

Proof. Using the $L^{1}$-contractivity of $\Phi$ we get

$$
\tau(|| h|a|)=\tau(|h a|) \geq \tau(|\Phi(h a)|)=\tau(|| \Phi(h)|\Phi(a)|), \quad a \in A .
$$

Taking the infimum over such an $a$ with $\Delta(\Phi(a)) \geq 1$, we obtain from Corollary 3.2, and Theorem 3.1 applied to $\mathcal{D}$, that $\Delta(h)=\Delta(|h|) \geq \Delta(|\Phi(h)|)=\Delta(\Phi(h))$.

We recall that although $L^{p}(M)$ is not a normed space if $1>p>0$, it is a socalled linear metric space with metric given by $\|x-y\|_{p}^{p}$ for any $x, y \in L^{p}$ (see [11, $4.9]$ ). Thus although the unit ball may not be convex, continuity still respects all elementary linear operations.

Corollary 3.4. Let $h \in L^{q}(M)_{+}$and $0<p, q<\infty$. If $h^{\frac{q}{p}} \in\left[h^{\frac{q}{p}} A_{0}\right]_{p}$, then $\Delta(h)=0$. Conversely, if $A$ is antisymmetric and $\Delta(h)=0$, then $h^{\frac{q}{p}} \in\left[h^{\frac{q}{p}} A_{0}\right]_{p}$. Indeed if $A$ is antisymmetric, then

$$
\Delta(h)=\inf \left\{\tau\left(\left|h^{\frac{q}{p}}\left(1-a_{0}\right)\right|^{p}\right)^{\frac{1}{q}}: a_{0} \in A_{0}\right\} .
$$

Proof. The first assertion follows by taking $a$ in the infimum in Corollary 3.2 to be of the form $1-a_{0}$ for $a_{0} \in A_{0}$.

If $A$ is antisymmetric, and if $t \geq 1$ with $\tau\left(\left|h^{\frac{q}{p}}\left(t 1+a_{0}\right)\right|^{p}\right)^{\frac{1}{q}}<\Delta(h)+\epsilon$, then $\tau\left(\left|h^{\frac{q}{p}}\left(1+a_{0} / t\right)\right|^{p}\right)^{\frac{1}{q}}<\Delta(h)+\epsilon$. From this last assertion, it follows that the infimums in Corollary 3.2 can be taken over terms of the form $1+a_{0}$ where $a_{0} \in A_{0}$. If this infimum were 0 , we could then find a sequence $a_{n} \in A_{0}$ with $h^{\frac{q}{p}}\left(1+a_{n}\right) \rightarrow 0$ with respect to $\|\cdot\|_{p}$. Thus $h^{\frac{q}{p}} \in\left[h^{\frac{q}{p}} A_{0}\right]_{p}$. 
Remark. The converse in the last result is false for general maximal subdiagonal algebras (e.g. consider $A=M=M_{n}$ ).

For a general maximal subdiagonal algebra $A$, and $h \in L^{1}(M)_{+}$we define $\delta(h)=$ $\inf \left\{\tau\left(\left|h^{\frac{1}{2}}\left(1-a_{0}\right)\right|^{2}\right): a_{0} \in A_{0}\right\}$. Note that $\delta(h)=\tau(h)$ in the example $M=A$.

We close this section with the following version of the Szegö formula valid for general positive linear functionals. Although the classical version of this theorem is usually attributed to Kolmogorov and Krein, we have been informed by Barry Simon that Verblunsky proved it first, in the mid 1930s (see e.g. [33]):

Theorem 3.5 (Noncommutative Szegö-Verblunsky-Kolmogorov-Krein theorem). Let $\omega$ be a positive linear functional on $M$, and let $\omega_{n}$ and $\omega_{s}$ be its normal and singular parts respectively, with $\omega_{n}=\tau(h \cdot)$ for $h \in L^{1}(M)_{+}$. If $\operatorname{dim}(\mathcal{D})<\infty$, then

$$
\Delta(h)=\inf \left\{\omega\left(|a|^{2}\right): a \in A, \Delta(\Phi(a)) \geq 1\right\} .
$$

The infimum remains unchanged if we also require $\Phi(a)$ to be invertible in $\mathcal{D}$.

Proof. Suppose that $\operatorname{dim}(\mathcal{D})<\infty$. All terminology and notation will be as in Lemma 3.2 of [ 6 , the preamble to the proof of the noncommutative F. \& M. Riesz theorem [6]. For the sake of clarity we pause to highlight the most important of these. If $\left(\pi_{\omega}, H_{\omega}, \Omega_{\omega}\right)$ is the GNS representation engendered by $\omega$, there exists a central projection $p_{0}$ in $\pi_{\omega}(M)^{\prime \prime}$ such that for any $\xi, \psi \in H_{\omega}$ the functionals $a \mapsto$ $\left\langle\pi_{\omega}(a) p_{0} \xi, \psi\right\rangle$ and $a \mapsto\left\langle\pi_{\omega}(a)\left(1-p_{0}\right) \xi, \psi\right\rangle$ on $M$ are respectively the normal and singular parts of the functional $a \mapsto\left\langle\pi_{\omega}(a) \xi, \psi\right\rangle$ [31, III.2.14]. In this representation $\Omega_{0}$ will denote the orthogonal projection of $\Omega_{\omega}$ onto the closed subspace $\overline{\pi_{\omega}\left(A_{0}\right) \Omega_{\omega}}$.

Let $d \in \mathcal{D}$ be given. We may of course select a sequence $\left(f_{n}\right) \subset A_{0}$ so that $\lim _{n \rightarrow \infty} \pi_{\omega}\left(f_{n}\right) \Omega_{\omega}=\Omega_{0}$. By the ideal property of $A_{0}$ and continuity, it then follows that

$$
\pi_{\omega}(d) \Omega_{0}=\lim _{n \rightarrow \infty} \pi_{\omega}\left(d f_{n}\right) \Omega_{\omega} \in \overline{\pi_{\omega}\left(A_{0}\right) \Omega_{\omega}} .
$$

Once again using the ideal property of $A_{0}$, the fact that $\Omega_{\omega}-\Omega_{0} \perp \overline{\pi_{\omega}\left(A_{0}\right) \Omega_{\omega}}$ now forces $\left\langle\pi_{\omega}(d)\left(\Omega_{\omega}-\Omega_{0}\right), \pi_{\omega}(a) \Omega_{\omega}\right\rangle=\left\langle\Omega_{\omega}-\Omega_{0}, \pi_{\omega}\left(d^{*} a\right) \Omega_{\omega}\right\rangle=0$ for every $a \in A_{0}$. Therefore

$$
\pi_{\omega}(d)\left(\Omega_{\omega}-\Omega_{0}\right) \perp \overline{\pi_{\omega}\left(A_{0}\right) \Omega_{\omega}} .
$$

From the facts in the previous two centered equations, it now follows that $\pi_{\omega}(d) \Omega_{0}$ is the orthogonal projection of $\pi_{\omega}(d) \Omega_{\omega}$ onto $\overline{\pi_{\omega}\left(A_{0}\right) \Omega_{\omega}}$. Using this fact we may now repeat the arguments of [6, Lemma $3.2(\mathrm{a}) \&(\mathrm{~b})(\mathrm{i})]$ for the functional

$$
\omega_{d}(\cdot)=\left\langle\pi_{\omega}(\cdot) \pi_{\omega}(d)\left(\Omega_{\omega}-\Omega_{0}\right), \pi_{\omega}(d)\left(\Omega_{\omega}-\Omega_{0}\right)\right\rangle
$$

to conclude that $\omega_{d}$ is normal, with $p_{0}\left(\pi_{\omega}(d)\left(\Omega_{\omega}-\Omega_{0}\right)\right)=\pi_{\omega}(d)\left(\Omega_{\omega}-\Omega_{0}\right)$, where $p_{0}$ is the central projection in $\pi_{\omega}(M)^{\prime \prime}$ mentioned above, and also: $p_{0} \pi_{\omega}(d)\left(\Omega_{\omega}-\Omega_{0}\right) \perp$ 
$p_{0} \pi_{\omega}\left(A_{0}\right) \Omega_{\omega}$ and $p_{0}\left(\pi_{\omega}(d) \Omega_{0}\right)$ is the orthogonal projection of $p_{0}\left(\pi_{\omega}(d) \Omega_{\omega}\right)$ onto $p_{0}\left(\overline{\pi_{\omega}\left(A_{0}\right) \Omega_{\omega}}\right)$. Thus we arrive at the fact that

$$
\begin{aligned}
\inf _{a \in A_{0}} \omega\left(|d+a|^{2}\right) & =\inf _{a \in A_{0}}\left\langle\pi_{\omega}(d) \Omega_{\omega}+\pi_{\omega}(a) \Omega_{\omega}, \pi_{\omega}(d) \Omega_{\omega}+\pi_{\omega}(a) \Omega_{\omega}\right\rangle \\
& =\inf _{a \in A_{0}}\left\|\pi_{\omega}(d) \Omega_{\omega}-\pi_{\omega}(a) \Omega_{\omega}\right\|^{2} \\
& =\left\langle\pi_{\omega}(d)\left(\Omega_{\omega}-\Omega_{0}\right), \pi_{\omega}(d)\left(\Omega_{\omega}-\Omega_{0}\right)\right\rangle \\
& =\left\langle p_{0} \pi_{\omega}(d)\left(\Omega_{\omega}-\Omega_{0}\right), p_{0} \pi_{\omega}(d)\left(\Omega_{\omega}-\Omega_{0}\right)\right\rangle \\
& =\inf _{a \in A_{0}}\left\langle p_{0} \pi_{\omega}(d) \Omega_{\omega}+p_{0} \pi_{\omega}(a) \Omega_{\omega}, p_{0} \pi_{\omega}(d) \Omega_{\omega}+p_{0} \pi_{\omega}(a) \Omega_{\omega}\right\rangle \\
& =\inf _{a \in A_{0}} \omega_{n}\left(|d+a|^{2}\right) \\
& =\inf _{a \in A_{0}} \tau\left(h|d+a|^{2}\right) .
\end{aligned}
$$

On taking the infimum over all $d \in \mathcal{D}$ with $\Delta(d) \geq 1$, the result follows from Corollary 3.2 ,

Remark. After seeing this last result, Xu was able to use our Szegö formula, and facts about singular states, to remove the hypothesis that $\operatorname{dim}(\mathcal{D})<\infty$, and to replace the ' 2 ' by a general $p$. See [2] for details.

\section{INNER-OUTER FACTORIZATION AND THE CHARACTERIZATION OF OUTERS}

Throughout this section $A$ is a maximal subdiagonal algebra. We recall that if $h \in H^{1}$, then $h$ is outer if $[h A]_{1}=H^{1}$. An inner element is a unitary which happens to be in $A$.

Lemma 4.1. Let $1 \leq p \leq \infty$. Then $h \in L^{p}(M)$ and $h$ is outer in $H^{1}$ iff $[h A]_{p}=$ $H^{p}$. (Note that $[\cdot]_{\infty}$ is the weak ${ }^{*}$ closure.)

If these hold, then $h \notin\left[h A_{0}\right]_{p}$.

Proof. It is obvious that if $[h A]_{p}=H^{p}$, then $[h A]_{1}=H^{1}$. Conversely, if $[h A]_{1}=H^{1}$ and $h \in L^{p}(M)$, then the first part of the proof of [5. Lemma 4.2] applied to $[h A]_{p}$ actually shows that $[h A]_{p}=[h A]_{1} \cap L^{p}(M)$ for all $1 \leq p \leq \infty$. Hence by [28, Proposition 2], we have

$$
[h A]_{p}=[h A]_{1} \cap L^{p}(M)=H^{1} \cap L^{p}(M)=H^{p} .
$$

If $h \in\left[h A_{0}\right]_{p}$, then $1 \in[h A]_{p} \subset\left[\left[h A_{0}\right]_{p} A\right]_{p} \subset\left[h A_{0}\right]_{p}$. Now $\Phi$ continuously extends to a map which contractively maps $L^{p}(M)$ onto $L^{p}(\mathcal{D})$ (see, e.g., Proposition 3.9 of [22]). If $h a_{n} \rightarrow 1$ in $L^{p}$, with $a_{n} \in A_{0}$, then

$$
\Phi\left(h a_{n}\right)=\Phi(h) \Phi\left(a_{n}\right)=0 \rightarrow \Phi(1)=1,
$$

This forces $\Phi(1)=0$, a contradiction.

Lemma 4.2. If $h \in H^{1}$ is outer, then as an unbounded operator $h$ has dense range and trivial kernel. Thus $h=u|h|$ for a unitary $u \in M$. Also, $\Phi(h)$ has dense range and trivial kernel.

Proof. If $h$ is considered as an unbounded operator, and if $p$ is the range projection of $h$, then since there exists a sequence $\left(a_{n}\right)$ in $A$ with $h a_{n} \rightarrow 1$ in the $L^{1}$-norm, we have that $p^{\perp}=0$. Thus the partial isometry $u$ in the polar decomposition of $h$ is 
an isometry, and hence is a unitary, in $M$. It follows that $|h|$ has dense range, and hence it, and $h$ also, have trivial kernel.

For the last part note that

$$
L^{1}(\mathcal{D})=\Phi\left(H^{1}\right)=\Phi\left([h A]_{1}\right)=[\Phi(h) \mathcal{D}]_{1} .
$$

Thus we can also apply the above arguments to $\Phi(h)$.

There is a natural equivalence relation on outers:

Proposition 4.3. If $h \in H^{p}$ is outer, and if $u$ is a unitary in $\mathcal{D}$, then $h^{\prime}=u h$ is also outer in $H^{p}$. If $h, k \in H^{p}$ are outer, then $|h|=|k|$ iff there is a unitary $u \in \mathcal{D}$ with $h=u k$. Such a $u$ is unique.

Proof. The first part is just as in the classical case. If $h, k \in H^{1}$ and $|h|=|k|$, then it follows, as in [30, p. 228], that $h=u k$ for a unitary $u \in M$ with $u, u^{*} \in H^{1}$. Thus $u \in H^{1} \cap M=A$ (see [28]), and similarly $u^{*} \in A$, and so $u \in \mathcal{D}$. The uniqueness of $u$ follows since the left support projection of an outer is 1 (see the proof of Lemma 4.2).

Remark. It follows that if $f=u h$ is a 'Beurling-Nevanlinna factorization' of an $f \in L^{p}(M)$, for a unitary $u \in M$ and $h$ outer in $H^{1}$, then this factorization is unique up to a unitary in $\mathcal{D}$. For if $u_{1} h_{1}=u_{2} h_{2}$ were two such factorizations, then $\left|h_{1}\right|=\left|u_{1} h_{1}\right|=\left|u_{2} h_{2}\right|=\left|h_{2}\right|$. By Proposition 4.3 we have $h_{1}=u h_{2}$ where $u \in \mathcal{D}$ is unitary. Since $u_{1} h_{1}=u_{1} u h_{2}=u_{2} h_{2}$, and since $h_{2}$ has dense range (see Lemma 4.2), we conclude that $u_{2}=u_{1} u$ and $h_{2}=u^{*} h_{1}$.

As in the classical case, if $h \in H^{2}$ is outer, then $h^{2}$ is outer in $H^{1}$. Indeed one may follow the proof on [30, p. 229], and the same proof shows that a product of any two outers is outer (see also the last lines of the proof of Theorem 4.4 below). We do not know whether every outer in $H^{1}$ is the square of an outer in $H^{2}$.

The first main theorem of the present section is a generalization of the beautiful classical characterization of outers in $H^{p}$ :

Theorem 4.4. Let $A$ be a subdiagonal algebra, let $1 \leq p \leq \infty$ and $h \in H^{p}$. If $h$ is outer, then $\Delta(h)=\Delta(\Phi(h))$. If $\Delta(h)>0$, this condition is also sufficient for $h$ to be outer.

Note that if $\operatorname{dim}(\mathcal{D})<\infty$, then $\Phi(h)$ will be invertible for any outer $h$ by Lemma 4.2. Thus in this case it is automatic that $\Delta(\Phi(h))>0$.

Proof. The case for general $p$ follows from the $p=1$ case and Lemma 4.1. Hence we may suppose that $p=1$.

First suppose that $h$ is outer. Given any $d \in L^{1}(\mathcal{D})$ and any $a_{0} \in\left[A_{0}\right]_{1}$, we clearly have that $\tau\left(\left|d-a_{0}\right|\right) \geq \tau\left(|d|-u^{*} a_{0}\right)=\tau(|d|)$, where $u$ is the partial isometry in the polar decomposition of $d$. In other words, for any $a \in[A]_{1}$ we have

$$
\|\Phi(a)\|_{1}=\inf _{a_{0} \in A_{0}}\left\|a-a_{0}\right\|_{1}=\inf _{a_{0} \in\left[A_{0}\right]_{1}}\left\|a-a_{0}\right\|_{1} .
$$

Therefore

$$
\tau(|\Phi(h) \widetilde{d}|)=\inf _{a_{0} \in A_{0}} \tau\left(\left|h \widetilde{d}-a_{0}\right|\right), \quad \widetilde{d} \in \mathcal{D} .
$$

Notice that $\left[h A_{0}\right]_{1}=\left[[h A]_{1} A_{0}\right]_{1}=\left[[A]_{1} A_{0}\right]_{1}=\left[A_{0}\right]_{1}$. Thus the above equality may alternatively be written as

$$
\tau(|\Phi(h) \widetilde{d}|)=\inf _{a_{0} \in A_{0}} \tau\left(\left|h \widetilde{d}-h a_{0}\right|\right), \quad \widetilde{d} \in \mathcal{D} .
$$


Finally notice that $|\Phi(h) \widetilde{d}|=|| \Phi(h)|\widetilde{d}|$ and $\left|h\left(\widetilde{d}-a_{0}\right)\right|=|| h\left|\left(\widetilde{d}-a_{0}\right)\right|$. Therefore if we now take the infimum over all $\widetilde{d} \in \mathcal{D}$ with $\Delta(\widetilde{d}) \geq 1$, Szegö's theorem will force

$$
\Delta(\Phi(h))=\Delta(|\Phi(h)|)=\Delta(|h|)=\Delta(h) .
$$

Next suppose that $\Delta(h)=\Delta(\Phi(h))>0$. We will first consider the case $h \in[A]_{2}$. By Lemma 4.1 we then need only show that $h$ is outer with respect to $[A]_{2}$. Replace $h$ by $\widetilde{h}=u^{*} h$ where $u$ is the partial isometry in the polar decomposition of $\Phi(h)$. If we can show that $\widetilde{h}$ is outer, it (and hence also $u^{*}$ ) will have dense range, which would force $u^{*}$ to be a unitary. Thus $h=u \widetilde{h}$ would then also be outer. Now notice that by construction $|h| \geq|\widetilde{h}|$ and $\Phi(\widetilde{h})=|\Phi(h)|$. From this and the generalized Jensen inequality we have

$$
\Delta(h)=\Delta(|h|) \geq \Delta(|\widetilde{h}|)=\Delta(\widetilde{h}) \geq \Delta(\Phi(\widetilde{h}))=\Delta(\Phi(h))=\Delta(h) .
$$

Thus $\Delta(\widetilde{h})=\Delta(\Phi(\widetilde{h}))>0$. We may therefore safely pass to the case where $\Phi(h) \geq 0$. By multiplying with a scaling constant, we may also clearly assume that $\Delta(h)=1$.

For any $d \in \mathcal{D}$ and $a \in A_{0}$ we have

$$
\tau\left(|1-h(d+a)|^{2}\right)=\tau\left(1-\Phi(h) d-d^{*} \Phi(h)\right)+\tau\left(|h(d+a)|^{2}\right) .
$$

To see this, simply combine the fact that $\tau \circ \Phi=\tau$ with the observation that $\Phi(h(d+a))=\Phi(h) \Phi(d+a)=\Phi(h) d$. With $d, a$ as above, notice that $\tau\left(|h(d+a)|^{2}\right)=$ $\tau\left(|| h|(d+a)|^{2}\right)$. By Szegö's theorem in the form of Corollary 3.2, we may select sequences $\left(d_{n}\right) \subset\left\{d \in \mathcal{D}^{-1}: \Delta(d) \geq 1\right\},\left(a_{n}\right) \subset A_{0}$, such that

$$
\lim _{n \rightarrow \infty} \tau\left(\left|h\left(d_{n}+a_{n}\right)\right|^{2}\right)=\Delta\left(|h|^{2}\right)=\Delta(h)^{2}=1 .
$$

Claim: We may assume the $d_{n}$ 's to be positive. To see this, notice that the invertibility of the $d_{n}$ 's means that for each $n$ we can find a unitary $u_{n} \in \mathcal{D}$ so that $d_{n} u_{n}=\left|d_{n}^{*}\right|$. Since for each $n$ we have

$$
\tau\left(\left|h\left(d_{n}+a_{n}\right)\right|^{2}\right)=\tau\left(\left|h\left(d_{n}+a_{n}\right) u_{n}\right|^{2}\right)=\tau\left(\left|h\left(\left|d_{n}^{*}\right|+a_{n} u_{n}\right)\right|^{2}\right),
$$

the claim follows. Notice that then $\tau\left(\Phi(h) d_{n}\right)=\tau\left(d_{n}^{1 / 2} \Phi(h) d_{n}^{1 / 2}\right) \geq 0$. Using in turn the $L^{2}$-contractivity of $\Phi$, the fact that $\Phi\left(h\left(d_{n}+a_{n}\right)\right)=\Phi(h) d_{n}$, and Hölder's inequality, we conclude that

$$
\tau\left(\left|h\left(d_{n}+a_{n}\right)\right|^{2}\right) \geq \tau\left(\left|\Phi(h) d_{n}\right|^{2}\right) \geq \tau\left(\left|\Phi(h) d_{n}\right|\right)^{2} \geq \tau\left(\Phi(h) d_{n}\right)^{2} \geq \Delta(\Phi(h))^{2}=1 .
$$

Since $\lim _{n \rightarrow \infty} \tau\left(\left|h\left(d_{n}+a_{n}\right)\right|^{2}\right)=1$, we must therefore also have $\lim _{n \rightarrow \infty} \tau\left(\Phi(h) d_{n}\right)$ $=1$. But if this is the case, then equation (4.1) assures us that $h\left(d_{n}+a_{n}\right) \rightarrow 1$ in the $L^{2}$-norm as $n \rightarrow \infty$. That is, $1 \in[h A]_{2}$. Clearly $h$ must then be outer.

Now let $h \in[A]_{1}$. By noncommutative Riesz factorization (see [22]) we may select $h_{1}, h_{2} \in[A]_{2}$ so that $h=h_{1} h_{2}$. Since $\Delta\left(h_{1}\right) \Delta\left(h_{2}\right)=\Delta(h)=\Delta(\Phi(h))=$ $\Delta\left(\Phi\left(h_{1}\right)\right) \Delta\left(\Phi\left(h_{2}\right)\right)>0$ and $\Delta\left(h_{i}\right) \geq \Delta\left(\Phi\left(h_{i}\right)\right)$ for each $i=1,2$ (by the generalized Jensen inequality), we must have $\Delta\left(h_{i}\right)=\Delta\left(\Phi\left(h_{i}\right)\right)$ for each $i=1,2$. Thus both $h_{1}$ and $h_{2}$ must be outer elements of $[A]_{2}$. Consequently

$$
[h A]_{1}=\left[h_{1} h_{2} A\right]_{1}=\left[h_{1}\left[h_{2} A\right]_{2}\right]_{1}=\left[h_{1}[A]_{2}\right]_{1}=\left[\left[h_{1}[A]_{2}\right]_{2}\right]_{1}=\left[[A]_{2}\right]_{1}=[A]_{1},
$$

so that $h$ is outer as required. 
Remark. In the general nonantisymmetric case, one can have outers with $\Delta(h)=0$. Indeed in the case that $A=M=L^{\infty}[0,1]$, then outer functions in $L^{2}$ are exactly the ones which are a.e. nonzero. One can easily find an increasing function $h$ : $[0,1] \rightarrow(0,1]$ satisfying $\Delta(h)=0$, or equivalently $\int_{0}^{1} \log h=-\infty$. See also [23]. This prompts the following:

Definition. We say that $h$ is strongly outer if it is outer and $\Delta(h)>0$.

Note that if $\operatorname{dim}(\mathcal{D})<\infty$, then every outer $h$ is strongly outer.

Corollary 4.5. Let $1 \leq p, q, r \leq \infty$ with $\frac{1}{p}=\frac{1}{q}+\frac{1}{r}$ and let $h=h_{1} h_{2}$ with $h_{1} \in H^{q}$ and $h_{2} \in H^{r}$. If $\Delta(h)>0$, then $h$ is outer in $H^{r}$ iff both $h_{1}$ and $h_{2}$ are outer.

Proof. The proof is clear from the last theorem, results in Section 2, and the generalized Jensen inequality (as in the last paragraph of the proof of the last theorem).

By the Riesz factorization mentioned in the introduction, any $h \in H^{r}$ is a product of the form in the last result.

Corollary 4.6. If $f \in L^{p}(\mathcal{D})$ with $\Delta(f)>0$, then $f$ is outer. Indeed there exist $d_{n} \in \mathcal{D}$ with $\Delta\left(f d_{n}\right) \geq 1$, and $f d_{n} \rightarrow 1$ in the 2-norm. Also, any $f \in L^{p}(M)$ with $\Delta(f)>0$ has left and right support projections equal to 1 . That is, as an unbounded operator it is one-to-one and has dense range.

Proof. For the first assertion note that $\Phi(f)=f$ and so $\Delta(f)=\Delta(\Phi(f))>0$. An inspection of the proof of the theorem gives the $d_{n}$ with the asserted properties. Thus $f$ clearly has left support projection 1 , and by symmetry the right projection is 1 too. Finally note that for the last assertion we may assume that $M=\mathcal{D}$.

Corollary 4.7. If $1 \leq p \leq \infty$ and $\Delta(h)>0$, then $h$ is outer in $H^{p}$ iff $[A h]_{p}=H^{p}$.

Proof. Replacing $A$ by $A^{*}$, it is trivial to see that $\Delta(h)=\Delta(\Phi(h))>0$ is equivalent to $\Delta\left(h^{*}\right)=\Delta\left(\Phi\left(h^{*}\right)\right)>0$. The latter is equivalent to $h^{*}$ being outer in $H^{2}\left(A^{*}\right)=$ $\left(H^{2}\right)^{*}$; or equivalently, to $\left(H^{2}\right)^{*}=\left[h^{*} A^{*}\right]_{2}$. Taking adjoints again gives the result.

Remark. The last result has the consequence that the theory has a left-right symmetry; for example, our inner-outer factorizations $f=u h$ below may instead be done with $f=h u$ (a different $u, h$ of course).

The following is a variant of Theorem 4.4 ;

Proposition 4.8. If $h \in H^{2}$, then $h$ is outer iff the wandering subspace of $[h A]_{2}$ (see [24, 7]) has a separating cyclic vector for the $\mathcal{D}$ action, and

$$
\|\Phi(h)\|^{2}=\inf \left\{\tau\left(\left|h\left(1-a_{0}\right)\right|^{2}\right): a_{0} \in A_{0}\right\} .
$$

Proof. (Following [23].) For $x \in L^{1}(M) \operatorname{set} \delta(x)=\inf \left\{\tau\left(\left.\left.|| x\right|^{\frac{1}{2}}\left(1-a_{0}\right)\right|^{2}\right): a_{0} \in A_{0}\right\}$.

First suppose that $h \in H^{2}$ is outer. Then $[h A]_{2} \ominus\left[h A_{0}\right]_{2}=H^{2} \ominus\left[A_{0}\right]_{2}=L^{2}(\mathcal{D})$, which has a separating cyclic vector. We next prove that if $h \in H^{2}$ is outer, then $\|\Phi(h)\|^{2}=\delta\left(|h|^{2}\right)$. To do this we view $\Phi$ as the orthogonal projection from $L^{2}(M)$ onto $L^{2}(\mathcal{D})$, which restricts to the orthogonal projection $P$ from $[A]_{2}$ onto $L^{2}(\mathcal{D})$. For any orthogonal projection $P$ from a Hilbert space onto a subspace $K$, we have $\|P(\zeta)\|=\inf \left\{\|\zeta-\eta\|: \eta \in K^{\perp}\right\}$. Thus $\|\Phi(h)\|^{2}=\inf \left\{\tau\left(\left|h-a_{0}\right|^{2}\right): a_{0} \in\left[A_{0}\right]_{2}\right\}$. 
Since $h$ is outer, we have $\left[[h A]_{2} A_{0}\right]_{2}=\left[H^{2} A_{0}\right]_{2}$, or $\left[h A_{0}\right]_{2}=\left[A_{0}\right]_{2}$. Thus $\|\Phi(h)\|^{2}=$ $\inf \left\{\tau\left(\left|h-h a_{0}\right|^{2}\right): a_{0} \in A_{0}\right\}=\delta\left(|h|^{2}\right)$.

Conversely, suppose that the wandering subspace of $[h A]_{2}$ has a separating cyclic vector. By [5, Corollary 1.2], we have $[h A]_{2}=u H^{2}$ for a unitary $u \in[h A]_{2} \subset H^{2}$. We have $h=u k$, with $k \in H^{2}$, and $[k A]_{2}=u^{*}[h A]_{2}=H^{2}$. So $k$ is outer. If $\|\Phi(h)\|^{2}=\delta(h)$, then using the notation in the last paragraph,

$$
\|\Phi(u) \Phi(k)\|^{2}=\delta\left(|u k|^{2}\right)=\delta\left(|k|^{2}\right)=\|\Phi(k)\|^{2} .
$$

That is, $\tau\left(\Phi(k)^{*}\left(1-\Phi(u)^{*} \Phi(u)\right) \Phi(k)\right)=0$. Since by Lemma 4.2 the left support projection of $\Phi(k)$ is 1, the functional $a \rightarrow \tau\left(\Phi(k)^{*} a \Phi(k)\right)$ is faithful on $M$ (indeed, $\tau\left(\Phi(k)^{*} a \Phi(k)\right) \neq 0$ for any nonzero $\left.a \in M_{+}\right)$, which forces $\Phi(u)^{*} \Phi(u)=1$. A simple computation shows that $\Phi\left(|u-\Phi(u)|^{2}\right)=1-\Phi(u)^{*} \Phi(u)=0$, so that $u=\Phi(u) \in \mathcal{D}$. Thus $h=u k$ is outer.

A classical theorem of Riesz-Szegö states that if $f \in L^{1}$ with $f \geq 0$, then $\int \log f>-\infty$ iff $f=|h|$ for an outer $h \in H^{1}$ iff $f=|h|^{2}$ for an outer $h \in H^{2}$. We now turn to this issue in the noncommutative case.

In what follows we are adapting ideas of Helson-Lowdenslager and Hoffman:

Lemma 4.9. Suppose that $A$ is a maximal subdiagonal algebra, and that $k \in L^{2}(M)$ with $k \notin\left[k A_{0}\right]_{2}$. Let $v$ be the orthogonal projection of $k$ onto $\left[k A_{0}\right]_{2}$. Then $|k-v|^{2}=$ $\Phi\left(|k-v|^{2}\right) \in L^{1}(\mathcal{D})$. Also, $\Delta(|k-v|) \geq \Delta(k)$.

Proof. Suppose that $k a_{n} \rightarrow v$, with $a_{n} \in A_{0}$. Clearly $k-v \perp k\left(1-a_{n}\right) a_{0} \in k A_{0}$ for all $a_{0} \in A_{0}$. In the limit, $k-v \perp(k-v) a_{0}$. That is, $\tau\left(|k-v|^{2} a_{0}\right)=0$, which by [4, $1.1 \& 4.1$ ] implies that $|k-v|^{2}=\Phi\left(|k-v|^{2}\right) \in L^{1}(\mathcal{D})$.

For the last assertion, note that by Lemma 3.1 we have $\Delta\left(|k-v|^{2}\right)=$ $\inf \left\{\tau\left(|(k-v) d|^{2}\right): d \in \mathcal{D}_{+}\right.$with $\left.\Delta(d) \geq 1\right\}$. But since $v d \in\left[k A_{0}\right]_{2}$ for every $d \in \mathcal{D}$, we may apply Szegö's theorem to conclude that this infimum majorises

$$
\inf \left\{\tau\left(\left|k d-k a_{0}\right|^{2}\right): d \in \mathcal{D}_{+} \text {with } \Delta(d) \geq 1, a_{0} \in A_{0}\right\}=\Delta\left(|k|^{2}\right)=\Delta(k)^{2},
$$

using the fact that $\left|k d-k a_{0}\right|=|| k\left|\left(d-a_{0}\right)\right|$.

Theorem 4.10. Suppose that $A$ is a maximal subdiagonal algebra, and that $k \in$ $L^{2}(M)$. Let $v$ be the orthogonal projection of $k$ onto $\left[k A_{0}\right]_{2}$. If $\Delta(k)>0$, then $k$ has an (essentially unique) Beurling-Nevanlinna factorization $k=u h$, where $u$ is a unitary in $M$, and equals the partial isometry in the polar decomposition of $k-v$, and $h$ is strongly outer and equals $u^{*} k$. We also have $\Delta(k)=\Delta(k-v)$. If $|k-v|$ is bounded below, then $(k-v) d=u$ for some $d \in \mathcal{D}$.

Proof. By Corollary 3.4. $k \notin\left[k A_{0}\right]_{2}$. By the lemma, $|k-v|^{2} \in L^{1}(\mathcal{D})$. Let $u$ be the partial isometry in the polar decomposition of $k-v$. Since $\Delta(k-v) \geq \Delta(k)>0$ by the lemma, we deduce from Corollary 4.6 that $u$ is surjective, and hence is a unitary. In the case that $|k-v|$ is bounded below, let $d=|k-v|^{-1} \in \mathcal{D}_{+}$, and then $u=(k-v) d$. Let $h=u^{*} k \in L^{2}(M)$. We claim that $\tau\left(u^{*} k a_{0}\right)=0$ for all $a_{0} \in A_{0}$, so that $h=u^{*} k \in L^{2}(M) \ominus\left[A_{0}^{*}\right]_{2}=H^{2}$. To see this, let $e_{n}$ be the spectral projection of $|k-v|$ corresponding to the interval $[0,1 / n]$. Then by elementary spectral theory, and since $k-v=u|k-v|$, we have $1-e_{n}=|k-v| r$ for some $r \in \mathcal{D}$. (Take $r=g(|k-v|)$ where $g$ is $\frac{1}{t} \chi_{\left(\frac{1}{n}, \infty\right)}$.) Thus

$$
\tau\left(a_{0}^{*} k^{*} u\left(1-e_{n}\right)\right)=\tau\left(a_{0}^{*} k^{*}(k-v) r\right)=0,
$$


since $k a_{0} r^{*} \in\left[k A_{0}\right]_{2}$ and $k-v \perp\left[k A_{0}\right]_{2}$. On the other hand, by the Borel functional calculus it is clear that $e_{n} \rightarrow e$ strongly, where $e$ is the spectral projection of $|k-v|$ corresponding to $\{0\}$. Since $\Delta(|k-v|) \geq \Delta(k)>0$ by the lemma, it is easy to see by spectral theory that $e=0$ (this essentially corresponds to the fact that a positive function $f$ which is 0 on a nonnull set has $\left.\int \log f=-\infty\right)$. We conclude that $\tau\left(a_{0}^{*} k^{*} u e_{n}\right) \rightarrow 0$, and it follows that

$$
\tau\left(a_{0}^{*} k^{*} u\right)=\tau\left(a_{0}^{*} k^{*} u e_{n}\right)+\tau\left(a_{0}^{*} k^{*} u\left(1-e_{n}\right)\right)=0 .
$$

To see that $u^{*} k$ is outer, we will use the criterion in Theorem 4.4. We claim that $\Phi\left(u^{*} k\right)=|k-v|$. To see this, note that by the last paragraph we have $\tau\left(u^{*} x\right)=0$ for any $x \in\left[k A_{0}\right]_{2}$, and in particular for $x=v c$ for any $c \in \mathcal{D}$. We have

$$
\tau\left(\Phi\left(u^{*} k\right) c\right)=\tau\left(u^{*} k c\right)=\tau\left(u^{*}(k-v) c\right)=\tau(|k-v| c) .
$$

Since this holds for any $c \in \mathcal{D}$ we have $\Phi\left(u^{*} k\right)=|k-v|$. Thus we have by the generalized Jensen inequality (Corollary 3.3) that

$$
\Delta(k)=\Delta\left(u^{*} k\right) \geq \Delta\left(\Phi\left(u^{*} k\right)\right)=\Delta(|k-v|) \geq \Delta(k) .
$$

Hence $h=u^{*} k$ is outer by Theorem 4.4 .

The uniqueness follows from the remark after Proposition 4.3.

Corollary 4.11. Suppose that $A$ is a maximal subdiagonal algebra with $\mathcal{D}$ finite dimensional, and that $k \in L^{2}(M)$ with $\Delta(k)>0$. Let $v$ be the orthogonal projection of $k$ onto $\left[k A_{0}\right]_{2}$. Then $|k-v|$ is invertible, and all the conclusions of the previous theorem hold.

Proof. By the above, $|k-v| \in L^{1}(\mathcal{D})=\mathcal{D}$, and $\Delta(|k-v|) \geq \Delta(k)>0$. Thus $|k-v|$ is invertible since $\mathcal{D}$ is finite dimensional. The rest follows from the previous theorem.

We next give a refinement of the 'Riesz factorization' into a product of two $H^{2}$ functions:

Corollary 4.12. If $A$ is a maximal subdiagonal algebra with $\mathcal{D}$ finite dimensional, and if $f \in L^{1}(M)$ with $\Delta(f)>0$, then there exists an outer $h_{2} \in H^{2}$, an invertible $d \in \mathcal{D}$ with $\Delta(d)=\frac{1}{\sqrt{\Delta(f)}}$, and an $h_{1} \in\left[f A_{0}\right]_{1}$ such that $f-h_{1} \in L^{2}(M)$, and $f=\left(f-h_{1}\right) d h_{2}$. If also $f \in H^{1}$, then this can be arranged with $h_{1} \in H^{1}, \Phi\left(h_{1}\right)=0$, and $f-h_{1} \in H^{2}$.

Proof. Let $k=|f|^{\frac{1}{2}}$. By Corollary 3.4 we have $k \notin\left[k A_{0}\right]_{2}$. If $u, v$ are as in Theorem 4.10, and if $f=w|f|=w k^{2}$ is the polar decomposition of $f$, then

$$
f=(w k u)\left(u^{*} k\right)=(w k(k-v)) d h_{2}=\left(f-h_{1}\right) d h_{2},
$$

where $h_{2}=u^{*} k$ and $h_{1}=w k v$.

If $k a_{n} \rightarrow v$ in the $L^{2}$-norm, with $a_{n} \in A_{0}$, then $f a_{n}=w k^{2} a_{n} \rightarrow w k v$ in the $L^{1}$-norm. Thus $h_{1} \in\left[f A_{0}\right]_{1}$. Also, $f-h_{1}=w k u d^{-1} \in L^{2}(M)$ (recall that since $\mathcal{D}$ is finite dimensional, $\left.d^{-1}=|k-v| \in \mathcal{D}\right)$. If $f \in H^{1}$, then $h_{1} \in\left[f A_{0}\right]_{1} \subset H^{1}$, and $\Phi\left(h_{1}\right)=0$. So $f-h_{1} \in H^{1} \cap L^{2}(M) \subset L^{2}(M) \ominus\left[A^{*}\right]_{2}=H^{2}$. 
Corollary 4.13. If $A$ is a maximal subdiagonal algebra, and if $f \in L^{1}(M)$ with $\Delta(f)>0$, then there exists a strongly outer $h \in H^{1}$, and a unitary $u \in M$ with $f=u h$.

Proof. By the proof of Corollary 4.12, and in that notation, we have $f=w k u h_{2}$ for an outer $h_{2}$. Note that $w$ is a unitary, since $f$ has dense range (Corollary 4.6). Since $\Delta(w k u)=\Delta(k)>0$ (by facts in Section 2), we have by the last theorem that $w k u=U h_{1}$ for a unitary $U$ and strongly outer $h_{1} \in H^{2}$. Let $h=h_{1} h_{2}$.

Corollary 4.14. If $A$ is a maximal subdiagonal algebra and $f \in L^{p}(M)$, then $\Delta(f)>0$ iff $f=$ uh for a unitary $u$ and a strongly outer $h \in H^{p}$. Moreover, this factorization is unique up to a unitary in $\mathcal{D}$.

Proof. $(\Rightarrow)$ By Corollary 4.13 we obtain the factorization with outer $h \in H^{1}$. Since $|f|=|h|$ we have $h \in L^{p}(M) \cap H^{1}=H^{p}$ (using [28, Proposition 2]), and $\Delta(h)>0$. $(\Leftarrow)$ We have $\Delta(f)=\Delta(u) \Delta(h)>0$.

The uniqueness of the factorization was discussed after Proposition 4.3 .

Remark. The $u$ in the last result is necessarily in $[f A]_{p}$ (indeed if $h a_{n} \rightarrow 1$ with $a_{n} \in A$, then $\left.f a_{n}=u h a_{n} \rightarrow u\right)$.

Corollary 4.15. If $A$ is a maximal subdiagonal algebra, then $f \in H^{p}$ with $\Delta(f)>0$ iff $f=u h$ for an inner $u$ and a strongly outer $h \in H^{p}$. Moreover, this factorization is unique up to a unitary in $\mathcal{D}$.

Proof. Clearly $f$ is also in $H^{1}$. Then $u$ is necessarily in $[f A]_{p} \subset H^{1}$, by the last remark. So $u \in H^{1} \cap M=A$ (see [28]). Thus $u$ is 'inner' (i.e., is a unitary in $\left.H^{\infty}=A\right)$.

An obvious question is whether there are larger classes of subalgebras of $M$ besides subdiagonal algebras for which such classical factorization theorems hold. The following shows that, with a qualification, the answer to this is in the negative:

Proposition 4.16. Suppose that $A$ is a tracial subalgebra of $M$ in the sense of our previous papers, such that every $f \in L^{2}(M)$ with $\Delta(f)>0$ is a product $f=$ uh for a unitary $u$ and an outer $h \in[A]_{2}$. Then $A$ is a finite maximal subdiagonal algebra.

Proof. Suppose that $A$ is a tracial subalgebra of $M$ with this factorization property. We will show that $A$ satisfies the ' $L^{2}$-density' and the 'unique normal state extension' properties, which together were shown in [4 to imply that $A$ is subdiagonal. As in our previous papers, $A_{\infty}$ is the tracial algebra $A_{\infty}=M \cap[A]_{2}$ extending $A$. If $x \in M$ is strictly positive, then $\Delta(x)>0$ by e.g. Theorem 2.1 (2). So $x=u h$ for a unitary $u$ and $h \in H^{2}$. Clearly $h$ is bounded, so that $h \in A_{\infty}$, and $x=\left(x^{*} x\right)^{\frac{1}{2}}=|h|$. Also, $h^{-1} \in A_{\infty}$, since if $h a_{n} \rightarrow 1$, then $a_{n} \rightarrow h^{-1}$. Thus $A_{\infty}$ has the 'factorization' property and so is maximal subdiagonal [4]. Hence $A_{\infty}+A_{\infty}^{*}$, and therefore also $A+A^{*}$, is dense in $L^{2}(M)$. Next, suppose that $g \in L^{1}(M)_{+}$satisfies $\tau\left(g A_{0}\right)=0$. We need to show that $g \in L^{1}(\mathcal{D})_{+}$. Since $\tau\left((g+1) A_{0}\right)=0$, we can replace $g$ with $g+1$, if necessary, to ensure that $\Delta(g)>0$. Let $f=g^{\frac{1}{2}} \in L^{2}(M)$. Then $\Delta(f)>0$, $f \perp\left[f A_{0}\right]_{2}$, and by hypothesis $f=u h$ for an outer $h \in[A]_{2}$ and some unitary $u$ in $M$. Since $h=u^{*} f \perp u^{*}\left[f A_{0}\right]_{2}=\left[h A_{0}\right]_{2}=\left[A_{0}\right]_{2}$, and $h \in[A]_{2}$, it follows that $h \in[\mathcal{D}]_{2}$. Thus $g \in[\mathcal{D}]_{1}=L^{1}(\mathcal{D})$. This verifies the 'unique normal state extension' property of [4. 
The following generalizes [18, Theorem 5.9]:

Corollary 4.17. If $f \in L^{1}(M)_{+}$, then the following are equivalent:

(i) $\Delta(f)>0$,

(ii) $f=|h|^{p}$ for a strongly outer $h \in H^{p}$,

(iii) $f=|k|^{p}$ for $k \in H^{p}$ with $\Delta(\Phi(k))>0$.

Proof. (i) $\Rightarrow$ (ii) By a previous result, $\Delta\left(f^{\frac{1}{p}}\right)>0$, and so by the last result we have $f^{\frac{1}{p}}=u h$, where $h$ is outer in $H^{p}$, and $u$ is unitary. Thus $f=\left(f^{\frac{1}{p}} f^{\frac{1}{p}}\right)^{\frac{p}{2}}=$ $\left(h^{*} h\right)^{\frac{p}{2}}=|h|^{p}$.

(ii) $\Rightarrow$ (iii) This follows from Theorem 4.4

(iii) $\Rightarrow$ (i) If $f=|k|^{p}$ for $k \in H^{p}$ with $\Delta(\Phi(k))>0$, then $\Delta(f)=\Delta(k)^{p} \geq$ $\Delta(\Phi(k))^{p}>0$ by Theorem 2.1 and the generalized Jensen inequality.

Of course in the case that $\mathcal{D}$ is finite dimensional one can drop the word 'strongly' in the last several results. In particular, in the case that the algebra $A$ is antisymmetric, these results and their proofs are much simpler and are spelled out in our survey [7.

Question. Is there a characterization of outers in $H^{1}$ in terms of extremals, as in the deLeeuw-Rudin theorem of e.g. [17, pp. 139-142], or [13, pp. 137-139]?

\section{ACKNOWLEDGEMENTS}

We thank Barry Simon for helpful information on Szegö's theorem and its generalizations, and for pointing out Verblunsky's precedence to the result usually attributed to Kolmogorov and Krein. We thank W. B. Arveson for continual encouragement, Finally, we are grateful to Q. Xu for suggesting several years ago to look at the noncommutative variant of the Riesz-Szegö theorem; and also for many insightful and valuable comments on the first version of our paper. He together with Bekjan have recently continued the $H^{p}$ theory contained in the present paper, by extending it to values $0<p<1$. This, together with other very interesting related results of theirs, is contained in [2].

\section{ADDED IN PROOF}

We have noticed that subdiagonal algebras satisfying a strong hypothesis on the diagonal $\mathcal{D}$ have a unique predual. Recently this fact has been proved with no restrictions on $\mathcal{D}$ by $\mathrm{Y}$. Ueda.

\section{REFERENCES}

[1] W. B. Arveson, Analyticity in operator algebras, Amer. J. Math. 89 (1967), 578-642. MR0223899 (36:6946)

[2] T. Bekjan and Q. Xu, Riesz and Szegö type factorizations for noncommutative Hardy spaces, Preprint (2006).

[3] D. P. Blecher and L. E. Labuschagne, Logmodularity and isometries of operator algebras, Trans. Amer. Math. Soc. 355 (2003), 1621-1646. MR.1946408(2004c:46113)

[4] D. P. Blecher and L. E. Labuschagne, Characterizations of noncommutative $H^{\infty}$, Integr. Equ. Oper. Theory 56 (2006), 301-321. MR2270840(2007m:46101) 
[5] D. P. Blecher and L. E. Labuschagne, A Beurling theorem for noncommutative $L^{p}$, To appear, J. Operator Theory (ArXiv: math.OA/0510358)

[6] D. P. Blecher and L. E. Labuschagne, Noncommutative function theory and unique extensions, Studia Math. 178 (2007), 177-195. MR2285438 (2007m:46102)

[7] D. P. Blecher and L. E. Labuschagne, Von Neumann algebraic $H^{p}$ theory, Proceedings of the 5th conference on function spaces, Contemp. Math., to appear, (ArXiV math.OA/0611879)

[8] L. G. Brown, Lidskiı's theorem in the type II case, Geometric methods in operator algebras (Kyoto, 1983), 1-35, Pitman Res. Notes Math. Ser., 123, Longman Sci. Tech., Harlow, 1986. MR866489 (88d:47024)

[9] A. Devinatz, The factorization of operator valued functions, Ann. of Math. 73 (1961), 458495. MR0126702 (23:A3997)

[10] R. Exel, Maximal subdiagonal algebras, Amer. J. Math. 110 (1988), 775-782. MR955297 (90b:46114)

[11] T. Fack and H. Kosaki, Generalized s-numbers of $\tau$-measurable operators, Pacific J. Math 123 (1986), 269-300. MR840845 (87h:46122)

[12] B. Fuglede and R. V. Kadison, Determinant theory in finite factors, Ann. of Math. 55 (1952), 520-530. MR0052696 (14:660a)

[13] T. W. Gamelin, Uniform Algebras, Second edition, Chelsea, New York, 1984.

[14] U. Haagerup and H. Schultz, Brown measures of unbounded operators affiliated with a finite von Neumann algebra, Math. Scand. 100 (2007), no. 2, 209-263. MR.2339369

[15] H. Helson, Lectures on invariant subspaces, Academic Press, New York-London, 1964. MR0171178 (30:1409)

[16] H. Helson and D. Lowdenslager, Prediction theory and Fourier series in several variables, Acta Math. 99 (1958), 165-202. MR0097688 (20:4155)

[17] K. Hoffman, Banach spaces of analytic functions, Dover (1988). MR.1102893 (92d:46066)

[18] K. Hoffman, Analytic functions and logmodular Banach algebras, Acta Math. 108 (1962), 271-317. MR0149330 (26:6820)

[19] M. Junge and D. Sherman, Noncommutative $L^{p}$-modules, J. Operator Theory 53 (2005), 3-34. MR2132686 (2006a:46077)

[20] L. E. Labuschagne, A noncommutative Szegö theorem for subdiagonal subalgebras of von Neumann algebras, Proc. Amer. Math. Soc., 133 (2005), 3643-3646. MR2163602 (2006d:46081)

[21] M. McAsey, P. Muhly, and K.-S. Saito, Nonselfadjoint crossed products (invariant subspaces and maximality), Trans. Amer. Math. Soc. 248 (1979), 381-409. MR.522266 (80j:46101b)

[22] M. Marsalli and G. West, Noncommutative $H^{p}$-spaces, J. Operator Theory 40 (1998), 339355. MR:1660390(2000b:46117)

[23] T. Nakazi, Extended weak-* Dirichlet algebras, Pacific J. Math. 81(1979), 493-513. MR.547616 (82a:46055)

[24] T. Nakazi and Y. Watatani, Invariant subspace theorems for subdiagonal algebras, J. Operator Theory 37 (1997), 379-395. MR 1452284 (98d:46059)

[25] E. Nelson, Notes on noncommutative integration, J. Funct. Anal. 15 (1974), 103-116. MR0355628 (50:8102)

[26] G. Pisier and Q. Xu, Noncommutative $L^{p}$ spaces, in Vol 2. of Handbook on Banach spaces, Ed. W. B. Johnson and J. Lindenstrauss, North-Holland, Amsterdam, 2003. MR1999201 (2004i:46095)

[27] G. Popescu, Entropy and multivariable interpolation, Mem. Amer. Math. Soc. 184(2006), No. 868. MR2263661 (2007h:47016)

[28] K.-S. Saito, A note on invariant subspaces for finite maximal subdiagonal algebras, Proc. Amer. Math. Soc. 77 (1979), 348-352. MR545594 (81b:46078)

[29] L. M. Schmitt, The Radon-Nikodym theorem for $L^{p}$-spaces of $W^{*}$-algebras, Publ. RIMS. Kyoto. Univ. 22(1986), 1025 - 1034. MR879995 (88c:46074)

[30] T. P. Srinivasan and J-K. Wang, Weak*-Dirichlet algebras, In Function algebras, Ed. Frank T. Birtel, Scott Foresman and Co., 1966, 216-249. MR0198282 (33:6441)

[31] M. Takesaki, Theory of Operator Algebras I, Springer, New York, 1979. MR548728 (81e:46038)

[32] M. Terp, $L^{p}$ spaces associated with von Neumann algebras, Notes, Math. Institute, Copenhagen Univ. 1981. 
[33] S. Verblunsky, On positive harmonic functions (second paper), Proc. London Math. Soc. 40 (1936), 290-320.

[34] L. Zsido, Spectral and ergodic properties of the analytic generators, J. Approximation Theory 20 (1977), 77-138. MR0463974 (57:3912)

Department of Mathematics, University of Houston, Houston, Texas 77204-3008

E-mail address: dblecher@math.uh.edu

Department of Mathematical Sciences, P.O. Box 392, 0003 UNISA, South Africa

E-mail address: labusle@unisa.ac.za 